\title{
The relationship between repeat resection and overall survival in patients with glioblastoma: a time-dependent analysis
}

\author{
*Debra A. Goldman, MS, ${ }^{1}$ Koos Hovinga, MD, ${ }^{2,4}$ Anne S. Reiner, MPH, ${ }^{1}$ Yoshua Esquenazi, MD, ${ }^{2,3}$ \\ Viviane Tabar, MD, ${ }^{2}$ and Katherine S. Panageas, $\mathrm{DrPH}^{1}$ \\ Departments of ${ }^{1}$ Epidemiology \& Biostatistics and ${ }^{2}$ Neurosurgery, Memorial Sloan Kettering Cancer Center, New York, New \\ York; ${ }^{3}$ Vivian L. Smith Department of Neurosurgery, The University of Texas Health Science Center at Houston, Texas; and \\ ${ }^{4}$ Department of Neurosurgery, Slotervaart Ziekenhuis, Amsterdam, The Netherlands
}

\begin{abstract}
OBJECTIVE Previous studies assessed the relationship between repeat resection and overall survival (OS) in patients with glioblastoma, but ignoring the timing of repeat resection may have led to biased conclusions. Statistical methods that take time into account are well established and applied consistently in other medical fields. The goal of this study was to illustrate the change in the effect of repeat resection on OS in patients with glioblastoma once timing of resection is incorporated.
\end{abstract}

METHODS The authors conducted a retrospective study of patients initially diagnosed with glioblastoma between January 2005 and December 2014 who were treated at Memorial Sloan Kettering Cancer Center. Patients underwent at least 1 craniotomy with both pre- and postoperative MRI data available. The effect of repeat resection on OS was assessed with time-dependent extended Cox regression controlling for extent of resection, initial Karnofsky Performance Scale score, sex, age, multifocal status, eloquent status, and postoperative treatment.

RESULTS Eighty-nine (55\%) of 163 patients underwent repeat resection with a median time between resections of 7.7 months (range 0.5-50.8 months). Median OS was 18.8 months (95\% confidence interval [Cl] 16.3-20.5 months) from initial resection. When timing of repeat resection was ignored, repeat resection was associated with a lower risk of death (hazard ratio $[\mathrm{HR}] 0.62,95 \% \mathrm{Cl} 0.43-0.90, \mathrm{p}=0.01$ ); however, when timing was taken into account, repeat resection was associated with a higher risk of death ( $\mathrm{HR} 2.19,95 \% \mathrm{Cl} 1.47-3.28, \mathrm{p}<0.001)$.

CONCLUSIONS In this study, accounting for timing of repeat resection reversed its protective effect on OS, suggesting repeat resection may not benefit OS in all patients. These findings establish a foundation for future work by accounting for timing of repeat resection using time-dependent methods in the evaluation of repeat resection on OS. Additional recommendations include improved data capture that includes mutational data, development of algorithms for determining eligibility for repeat resection, more rigorous statistical analyses, and the assessment of additional benefits of repeat resection, such as reduction of symptom burden and enhanced quality of life.

https://thejns.org/doi/abs/10.3171/2017.6.JNS17393

KEY WORDS glioblastoma; proportional hazards model; repeat surgery; survival analysis; time-dependent model; oncology

$\mathrm{G}$ LIOBLASTOMA is the most common form of primary malignant brain tumors $(46 \%){ }^{26}$ The prognosis with glioblastoma is notoriously poor, with median survival from diagnosis between 14 and 18 months ${ }^{26}$ and a 5-year survival estimate of $5.1 \%$ (95\% confidence interval [CI] 4.8\%-5.4\%). ${ }^{26}$ Standard treatment regimens include surgery followed by radiation and chemotherapy with temozolomide. ${ }^{31}$ Maximal tumor resection is a challenge due to the infiltrative nature of these tumors, and consequently, tumors recur in nearly all patients. Repeat resection is a potential treatment option at recurrence, with the goals of reducing neurological symptoms, meeting

ABBREVIATIONS $\mathrm{Cl}=$ confidence interval; $\mathrm{EOR}=$ extent of resection; $\mathrm{HR}=$ hazard ratio; $\mathrm{HSRT}=$ hypofractionated stereotactic radiation therapy; $\mathrm{KM}=\mathrm{Kaplan}-\mathrm{Meier}$; KPS = Karnofsky Performance Scale; OS = overall survival.

SUBMITTED February 27, 2017. ACCEPTED June 30, 2017.

INCLUDE WHEN CITING Published online January 5, 2018; DOI: 10.3171/2017.6.JNS17393.

* Drs. Tabar and Panageas share senior authorship of this work. 
eligibility requirements for clinical trials, and extending overall survival (OS).

The vast body of literature has demonstrated that repeat resection results in an OS benefit for patients with glioblastoma, $, 3,4,7,8,10,18,19,24,29,32$ however, these studies only took into account repeat resection status, treating it as a standard baseline covariate, and failed to account for its timing. Both whether repeat resection occurred, and when it occurred, are required to evaluate the impact of repeat resection on OS. Failure to do so results in a survival bias, which can be illustrated by considering two patients who survived the same amount of time. The impact of repeat resection on OS for a patient who underwent repeat resection 3 months after diagnosis and lived 18 months total is not the same for a patient who had repeat resection at 17 months and lived another month. Yet, these patients would be treated identically if timing of repeat resection is ignored in the analysis. Repeat resection status is unknown at initial diagnosis and changes over the course of a patient's illness. Repeat resection is, therefore, a timedependent variable.

This survival bias and corresponding time-dependent methodology are well-established in the statistical literature and were initially addressed in the seminal works by Crowley and $\mathrm{Hu}^{12}$ and Mantel and $\mathrm{Byar}^{23}$ in the heart transplant setting. Furthermore, the importance of timedependent methods were shown in other cancer treatments, such as bone marrow transplant,$^{20}$ adjuvant therapy for breast cancer, ${ }^{21}$ and metformin use for pancreatic cancer. ${ }^{9}$ As demonstrated by these studies and by the study of Anderson et al., ${ }^{2}$ ignoring timing biases the findings in favor of patients who survive long enough or who are healthy enough to receive additional treatment. Beyersmann et al. ${ }^{5,6}$ mathematically illustrated that ignoring the timing of a time-dependent covariate will always overestimate any survival benefit or underestimate any survival risk. Although these methods are fully established in the statistical literature and adopted in some clinical fields, the lack of use in many studies persists, ${ }^{33}$ including in the glioblastoma repeat resection literature. Our goal was to incorporate time-dependent methodology in the glioblastoma repeat resection setting.

In this study, we assessed the relationship between repeat resection and OS in patients with glioblastoma accounting for the timing of repeat resection, and demonstrated how the results change if timing is ignored. We also provided approaches to graphically display this type of data.

\section{Methods}

\section{Study Population}

We reviewed a prospectively maintained neurosurgery database at Memorial Sloan Kettering Cancer Center, and identified patients who were newly diagnosed with glioblastoma between January 2005 and December 2014 and who underwent at least 1 craniotomy at diagnosis. The 2005-2014 time span was chosen as standard-of-care therapy and imaging guidelines were consistent between these dates, and to ensure we had sufficient follow-up. We collected survival data until August 2015. Our IRB ap- proved a waiver of informed consent for this retrospective, HIPAA-compliant study.

Initially, 509 cases were identified. Patients were excluded if their craniotomies occurred at outside institutions as imaging was frequently unavailable, they had lowgrade glioma prior to glioblastoma diagnosis, their surgery was only a biopsy, they received prior treatment for presumed brain metastasis that was eventually diagnosed as glioblastoma, or extent of resection (EOR) could not be derived (Fig. 1). Six patients received a novel agent as part of a phase I clinical trial 2 weeks prior to initial surgery. However, these patients remained in our cohort as the trial did not alter the timing of surgery and the patients were not assessed for response to the agent. Our final sample consisted of 163 patients.

\section{Data Collection}

Diagnostic, demographic, initial Karnofsky Performance Scale (KPS) score, treatment, pathology, and outcome records were extracted from the database. Two neurosurgical oncology fellows (K.H. and Y.E.) measured pre- and postoperative tumor volume on MRI. Volumes were collected when the preoperative scan was within 1 week of surgery and the postoperative scan was within 48 hours of surgery. Volumetric measurements were obtained using iPlan Net software (version 3.0.0, Brainlab AG). Regions of interest were manually drawn on each postcontrast axial T1-weighted MR image, and the contrast-enhanced tumor volume in cubic centimeters was computed. For initial and repeat resection, EOR was defined as the percentage change between preoperative and postoperative contrast-enhanced tumor volumes. As no clear consensus exists in the literature on a defined cutoff for grosstotal resection versus subtotal resection, we examined EOR continuously and using $97 \%{ }^{25}$ as a predefined cutoff. In addition to measuring volume, the two neurosurgical oncology fellows recorded whether the tumor was located in an eloquent region, which included primary motor and/ or speech areas, and if the tumor was multifocal. Eloquent areas included precentral gyrus, postcentral gyrus, inferior posterior left frontal lobe (Broca's area), left posterior superior temporal gyrus (Wernicke's area), and supramarginal and angular gyri. Multifocal lesions were defined as separate Gd-enhanced areas where a pattern of dissemination along an established route was observed.

KPS score at repeat resection was unavailable for most patients, methylation status was missing for approximately half, and $I D H-1$ status was missing for $75 \%$, so these factors were not included in analyses.

\section{Statistical Analyses}

OS was calculated from initial surgery until death or last follow-up date. Patients alive at last follow-up were censored. OS at 1 and 5 years was estimated using Kaplan-Meier (KM) methods. To provide median survival from second surgery, OS was also estimated from the time of repeat resection until death or last follow-up for patients who underwent repeat resection.

Our primary goal was to assess the relationship between repeat resection and OS applying time-dependent 
methods, which account for timing of repeat resection, and to show how our results change from an analysis that assumes repeat resection status was known at the time of initial resection (i.e., ignoring timing of repeat resection). To accomplish this, we used Cox proportional hazards regression, treating repeat resection (yes vs no) as a standard baseline factor, and then used extended Cox regression treating repeat resection as a time-dependent factor in univariable and multivariable analyses.

In addition to repeat resection in the time-dependent analyses, multifocal status, eloquent status, EOR, and postoperative chemotherapy and radiation after initial surgery were treated as time-dependent covariates, which meant their values at repeat resection were incorporated into the model, if applicable. Age at initial surgery, sex, and KPS score at initial surgery were treated as static covariates. We adjusted for factors in the multivariable analyses regardless of univariable significance. Three patients were missing initial KPS scores and were excluded from multivariable analysis.

Traditional KM curves are a beneficial visual tool for illustrating OS and differences in survival between groups; unfortunately, they only represent static covariates where the value is fixed at the start of the study. Repeat resection status is not known at the time of initial resection, so we used two methods, which modify the standard KM plots, to graphically illustrate the relationship between OS and repeat resection. We used the landmark method, ${ }^{2}$ which selects a predefined time point after the start of the study (in our case initial resection) to use as a landmark time. The KM curve then starts at this landmark time rather than at initial resection. If repeat resection occurred before this landmark time, the patient was considered to have repeat resection. If a patient did not have repeat resection by the landmark time they were considered not to have repeat resection. We chose two predefined time points: the approximate median (50th percentile) and 75th percentile of time between the first and second surgery, which were 8 months and 14 months, respectively. Two time points, as opposed to one, were used to assess the consistency of the curves. OS was then calculated from 8 and 14 months after initial surgery until death or last follow-up. This method allows for stratification similar to traditional KM curves, as the covariate value is fixed at the landmark time; however, it has limitations as it does not consider patients who had repeat resection after the landmark time, and it excludes patients who die or are censored prior to the landmark time.

An alternative method proposed by Simon and Makuch $^{30}$ includes all patients, but does not consider a patient's value to be fixed. In Simon and Makuch's method, patients move from the group of "no resection" to the group of "repeat resection" at the time their repeat resection occurs. Therefore, the number of patients in each risk set fluctuates over time. These graphs do not allow for inference, but help visualize the effect of a time-dependent covariate on survival. We presented this modified plot next to the traditional KM curve in which timing of repeat resection was not taken into account, to illustrate the differences.

In post hoc analyses, we assessed demographic and

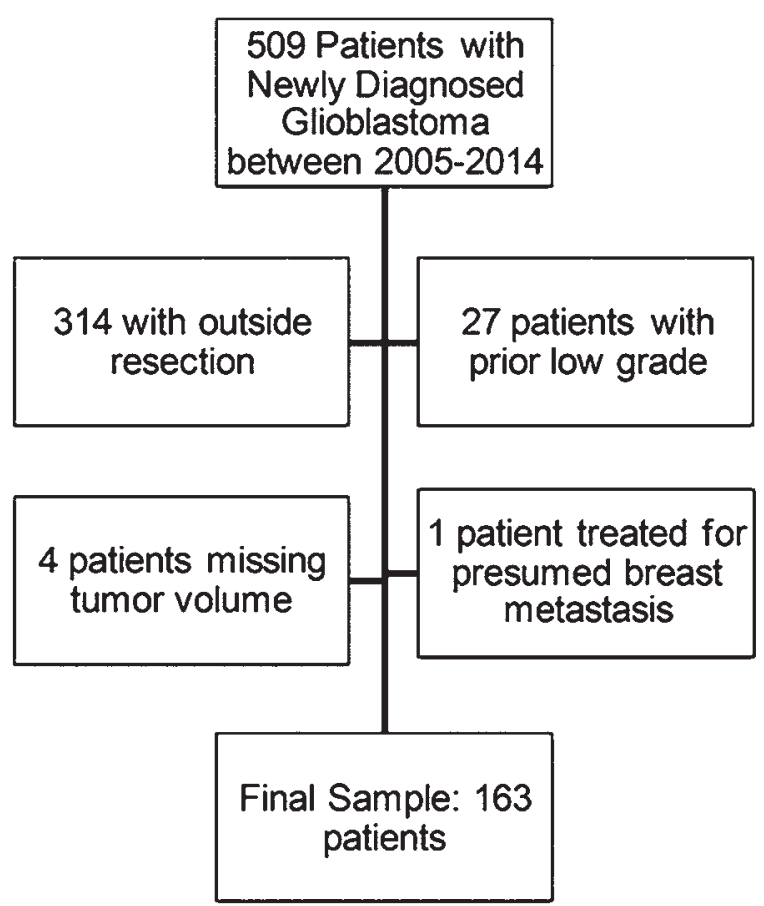

FIG. 1. Flow chart for patient sample selection. Our initial sample consisted of 509 patients and patients were excluded if they had an outside resection $(n=314)$, if tumor volume was unavailable $(n=4)$, if they had a prior low-grade glioma $(n=27)$, or if they were incorrectly treated for a presumed metastasis that turned out to be glioblastoma $(n=1)$. The most common reason for exclusion was outside resection.

clinical differences between those with repeat resection and those without, using competing risks analysis with Fine and Gray methods ${ }^{14}$ treating death as a competing risk. We considered 2 -sided $\mathrm{p}$ values $<0.05$ to be statistically significant. The proportional hazards assumption for the extended Cox model was checked with Schöenfeld residuals and log-log plots. All analyses were performed using SAS (version 9.4, SAS Institute) and Stata (version 13.1).

\section{Results}

\section{Patient and Treatment Characteristics}

One hundred sixty-three patients were included with a median age of 62 years (range $20-83$ years; Table 1 ). The majority of patients were male $(111 / 163,68 \%)$ and white $(153 / 163,94 \%)$. Eighty-nine patients $(55 \%)$ had a repeat resection within a median 7.7 months (range 0.5-50.8 months) from initial surgery. The 30-day postoperative mortality rate was $1 \%(2 / 163)$ for initial surgery and $1 \%$ $(1 / 89)$ for repeat resection.

The median pre- and postoperative volumes were 30.2 $\mathrm{cm}^{3}$ (range 1.2-135.7 $\mathrm{cm}^{3}$ ) and $1.80 \mathrm{~cm}^{3}$ (range 0-78.0 $\mathrm{cm}^{3}$ ), respectively, resulting in a median EOR of $92.9 \%$ (range 3.8\%-100\%). At initial surgery, 56\% (91/163) of patients had tumors in eloquent regions and 13\% (21/163) had multifocal tumors. After initial surgery, the majority of patients received radiation therapy $(142 / 163,87 \%)$ and chemotherapy $(142 / 163,87 \%)$. Most of the patients $(141 / 163,87 \%)$ received temozolomide (Table 2$)$. 
TABLE 1. Patient characteristics

\begin{tabular}{|c|c|}
\hline Variable & No. $(\%)$ \\
\hline No. of patients & 163 \\
\hline Median age at initial surgery in yrs (range) & $62.4(20.1-82.9)$ \\
\hline \multicolumn{2}{|l|}{ Sex } \\
\hline Male & $111(68)$ \\
\hline Female & $52(32)$ \\
\hline \multicolumn{2}{|l|}{ Race } \\
\hline Unknown & $3(2)$ \\
\hline White & $153(94)$ \\
\hline Asian & $5(3)$ \\
\hline Black & $2(1)$ \\
\hline \multicolumn{2}{|l|}{ Initial KPS score } \\
\hline \multicolumn{2}{|l|}{ Continuous } \\
\hline Median (range) ${ }^{*}$ & $90(30-100)$ \\
\hline \multicolumn{2}{|l|}{ Count } \\
\hline Missing & $3(2)$ \\
\hline 30 & $1(1)$ \\
\hline 50 & $1(1)$ \\
\hline 60 & $9(5)$ \\
\hline 70 & $21(13)$ \\
\hline 80 & $44(27)$ \\
\hline 90 & $64(39)$ \\
\hline 100 & $20(12)$ \\
\hline \multicolumn{2}{|l|}{ Repeat resection } \\
\hline No & $74(45)$ \\
\hline Yes & $89(55)$ \\
\hline Median mos btwn 1st \& 2nd surgery (range) $\dagger$ & $7.7(0.5-50.8)$ \\
\hline \multicolumn{2}{|l|}{ Vital status } \\
\hline Alive & $33(20)$ \\
\hline Dead & $130(80)$ \\
\hline \multicolumn{2}{|l|}{ MGMT status at initial surgery } \\
\hline Unknown & $58(36)$ \\
\hline Unmethylated & $74(45)$ \\
\hline Methylated & $31(19)$ \\
\hline
\end{tabular}

MGMT $=0^{6}$-methylguanine-DNA methyltransferase.

All data given as number of patients (\%) unless otherwise specified.

* For 160 patients.

$\dagger$ For 89 patients.

Median tumor volume was $24.3 \mathrm{~cm}^{3}$ (range 2.4-104.9 $\mathrm{cm}^{3}$ ) prior to repeat resection and $3.4 \mathrm{~cm}^{3}$ (range $0-47.5$ $\mathrm{cm}^{3}$ ) after repeat resection, resulting in a median EOR of $84.2 \%$ (range $22.1 \%-100 \%$ ). At repeat resection, $48 \%$ of patients (43/89) had tumors in eloquent regions and $8 \%$ (7/89) had multifocal tumors.

\section{Overall Survival \\ Estimates}

At the end of follow-up, 130 patients (80\%) had died, with a median OS of 18.8 months (95\% CI 16.3-20.5 months). One- and 5-year OS estimates were 70.8\% (95\% CI $63.1 \%-77.3 \%)$ and $4.3 \%$ (95\% CI 1.2\%-10.4\%), respec-
TABLE 2. Treatment characteristics

\begin{tabular}{|c|c|}
\hline Treatment Characteristic & No. $(\%)$ \\
\hline \multicolumn{2}{|l|}{ Neoadjuvant } \\
\hline \multicolumn{2}{|l|}{ Received neoadjuvant chemotherapy } \\
\hline No & $157(96)$ \\
\hline Yes & $6(4)$ \\
\hline \multicolumn{2}{|l|}{ After initial surgery } \\
\hline \multicolumn{2}{|l|}{ Chemotherapy after initial surgery } \\
\hline No & $21(13)$ \\
\hline Yes & $142(87)$ \\
\hline $\begin{array}{l}\text { Median days btwn initial surgery and } \\
\text { chemotherapy (range) }\end{array}$ & $28.0(1-170)$ \\
\hline \multicolumn{2}{|l|}{ PostOp1 temozolomide } \\
\hline No & $22(13)$ \\
\hline Yes & $141(87)$ \\
\hline \multicolumn{2}{|l|}{ PostOp1 bevacizumab } \\
\hline No & $127(78)$ \\
\hline Yes & $36(22)$ \\
\hline \multicolumn{2}{|l|}{ PostOp1 carmustine } \\
\hline No & $156(96)$ \\
\hline Yes & $7(4)$ \\
\hline \multicolumn{2}{|l|}{ Radiation after initial surgery } \\
\hline No & $21(13)$ \\
\hline Yes & $142(87)$ \\
\hline $\begin{array}{l}\text { Median days btwn initial surgery and } \\
\text { radiation (range) }{ }^{*}\end{array}$ & $28.0(1-320)$ \\
\hline \multicolumn{2}{|l|}{ After second surgery† } \\
\hline \multicolumn{2}{|l|}{ Chemotherapy after second surgery } \\
\hline No & $31(35)$ \\
\hline Yes & $58(65)$ \\
\hline $\begin{array}{l}\text { Median days btwn second surgery and } \\
\text { chemotherapy (range) }\end{array}$ & $30.0(11-842)$ \\
\hline \multicolumn{2}{|l|}{ PostOp2 temozolomide } \\
\hline No & $57(64)$ \\
\hline Yes & $32(36)$ \\
\hline \multicolumn{2}{|l|}{ PostOp2 bevacizumab } \\
\hline No & $54(61)$ \\
\hline Yes & $35(39)$ \\
\hline \multicolumn{2}{|l|}{ PostOp2 carmustine } \\
\hline No & $75(84)$ \\
\hline Yes & $14(16)$ \\
\hline \multicolumn{2}{|l|}{ Radiation after second surgery } \\
\hline No & $78(88)$ \\
\hline Yes & $11(12)$ \\
\hline $\begin{array}{l}\text { Median days btwn second surgery and } \\
\text { radiation (range) } \ddagger\end{array}$ & $32.0(11-356)$ \\
\hline
\end{tabular}

PostOp1 = after initial surgery; PostOp2 = after repeat resection.

All data given as number of patients (\%) unless otherwise specified.

* For 142 patients.

$\dagger$ For 89 patients.

‡ For 11 patients. 

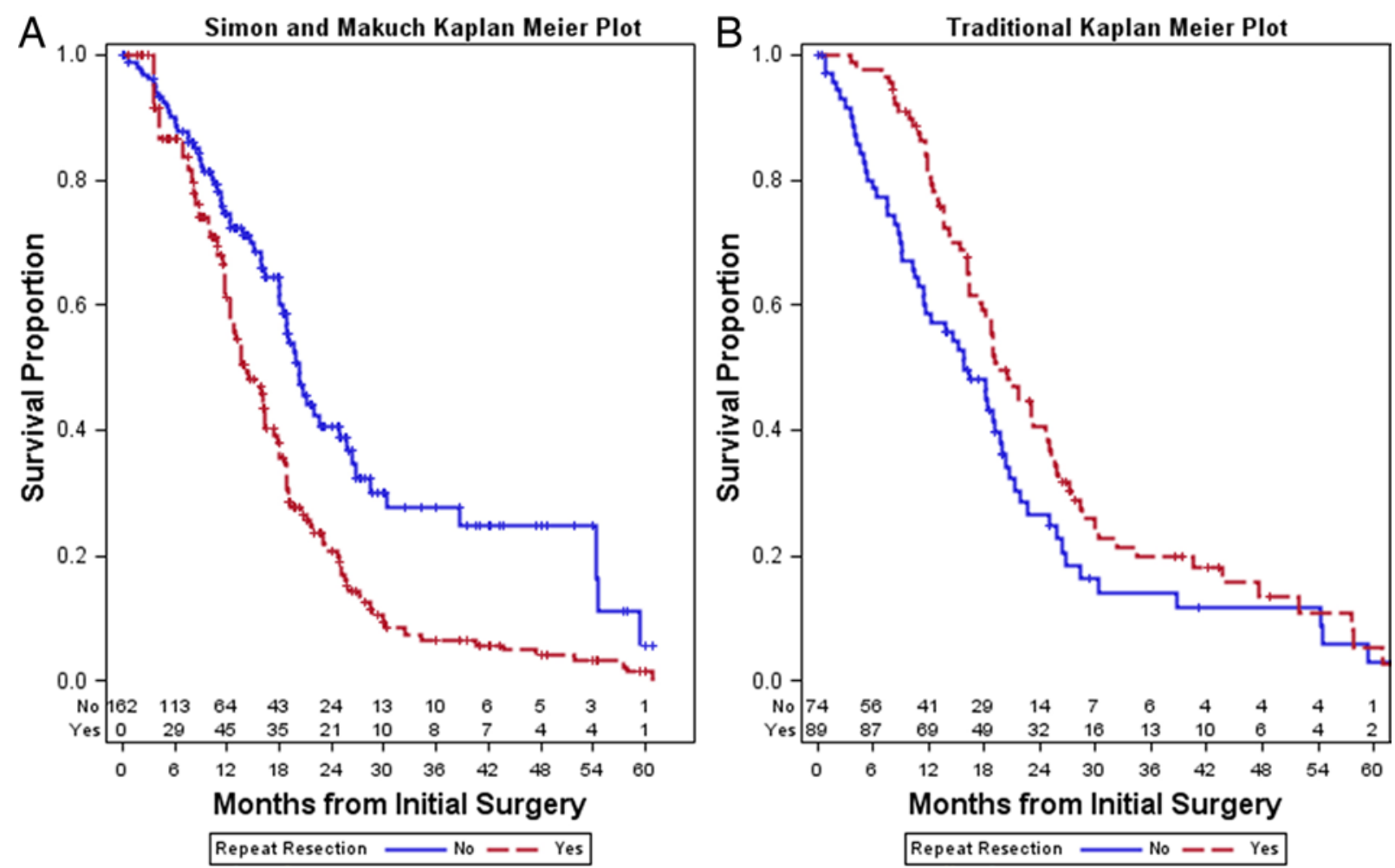

FIG. 2. A: Simon and Makuch modified KM plot. In this figure, patients moved from "no resection" to "repeat resection" if and when their repeat resection occurred. Survival probability was lower for patients who underwent repeat resection compared with those who did not. This figure is an illustrative tool for the formal time-dependent analysis and cannot be used to make inferences. B: Traditional KM plot. This figure illustrates the relationship between repeat resection and OS if one were to ignore timing of repeat resection and treat repeat resection as a fixed effect. Figure is available in color online only.

tively. In the 89 patients who underwent repeat resection, median OS from repeat resection was 9.0 months $(95 \% \mathrm{CI}$ 7.7-12.8 months).

\section{Univariable Analyses}

Prior to assessing repeat resection with time-dependent methods, we first treated it as a standard baseline covariate, ignoring timing. When timing of resection was ignored, repeat resection was associated with a lower risk of death (hazard ratio [HR] 0.70, 95\% CI 0.50-0.99, p = 0.04). After accounting for the timing of re-resection in a time-dependent model, repeat resection was associated with a higher risk of death (HR 2.02, 95\% CI 1.40-2.91, $\mathrm{p}<0.001)$.

Similarly, when repeat resection was treated as a static effect in the traditional KM plot (Fig. 2B), patients with repeat resection demonstrated better survival than patients without repeat resection. In contrast, when timing was incorporated with the Simon and Makuch method (Fig. $2 \mathrm{~A}$ ), patients with repeat resection demonstrated worse survival compared with those without repeat resection. The time-dependent univariable result was also consistent with the landmark curves in Fig. 3. With either landmark time point, the survival curve was lower for patients who had repeat resection by the landmark time compared with those without repeat resection by the landmark time.

In addition to repeat resection, in univariable analyses, those with multifocal tumors had a higher risk of death (HR 1.86, 95\% CI 1.14-3.03, $\mathrm{p}=0.01$ ) as did patients with tumors located in eloquent regions (HR 1.54, 95\% CI $1.08-2.20, p=0.02$; Table 3 ). Other significant predictors included EOR, in which increases in percentage tumor removed were associated with a lower risk of death (HR $0.15,95 \%$ CI $0.06-0.37, \mathrm{p}<0.001$ ), adjuvant chemotherapy (HR $0.30,95 \%$ CI $0.17-0.56, \mathrm{p}<0.001)$ and radiation therapy (HR 0.31, 95\% CI 0.17-0.56, p < 0.001), where those with treatment had a lower risk of death. Of note, the same pattern held for EOR when dichotomized (HR 0.59, $95 \%$ CI $0.39-0.88, p=0.009$ ) or when adjuvant treatment was combined (HR 0.54, 95\% CI 0.40-0.73, p < 0.001). Age (HR 1.03, 95\% CI 1.01-1.05, $\mathrm{p}=0.008)$ and sex (HR $0.67,95 \%$ CI $0.45-0.99, p=0.04)$ were also associated with OS, whereby older patients were at an increased risk of death and women had a lower risk of death. Higher initial KPS score was associated with a lower risk of death (HR $0.98,95 \%$ CI $0.97-1.00, p=0.03$ ) when treated as an ordinal covariate, but a significant association was not found when dichotomized into $\leq 70$ versus $>70$ (HR 1.25 , $95 \%$ CI $0.82-1.90, \mathrm{p}=0.30$ ).

\section{Multivariable Analyses}

Similar to the univariable analyses, we first treated repeat resection as a standard baseline covariate. When timing of resection was ignored, patients with repeat resection 

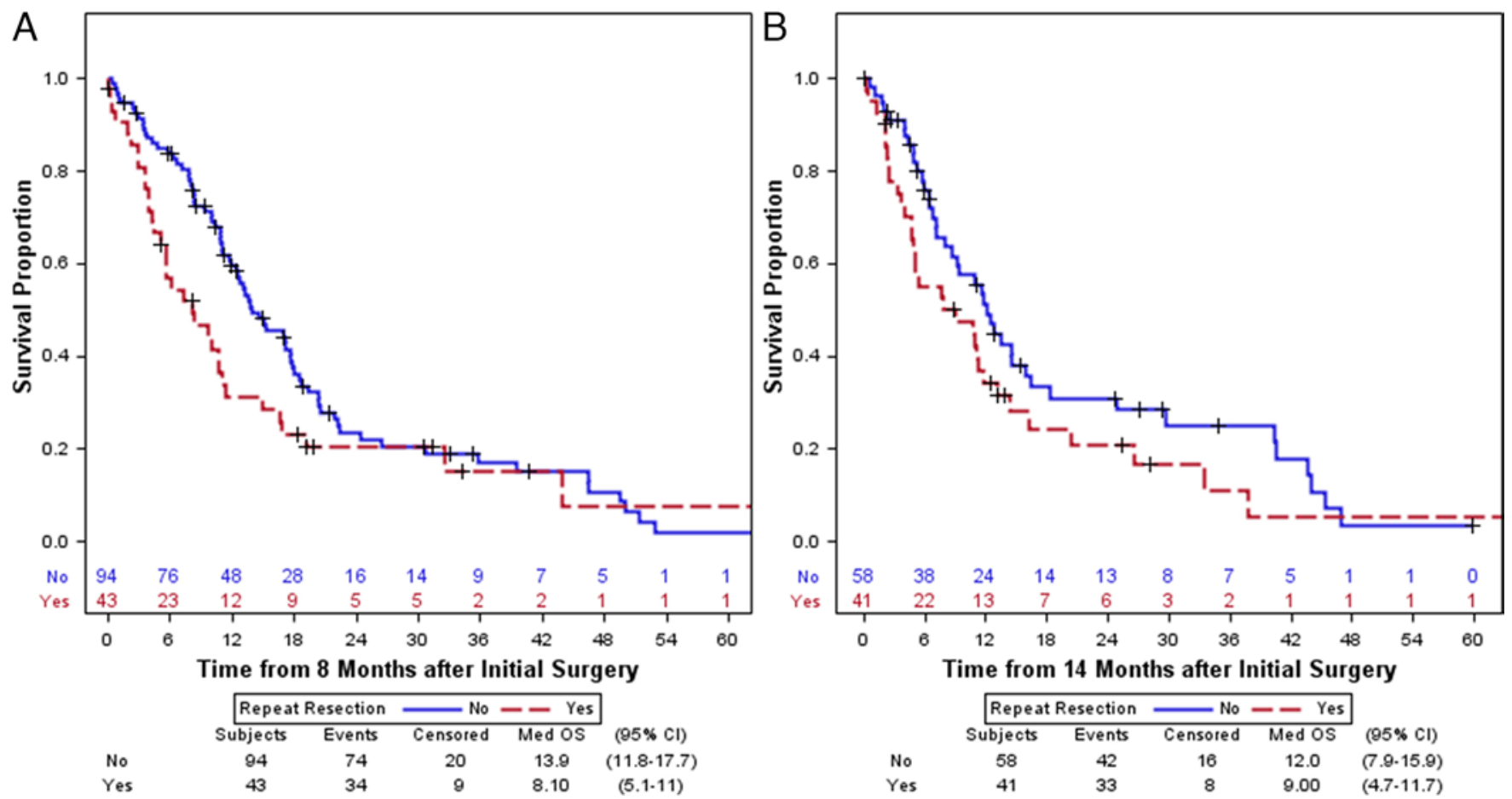

FIG. 3. KM survival plots landmarked at 8 (A) and 14 (B) months. Plots were drawn from 8 and 14 months from initial surgery, which were the approximate 50th and 75th percentile for time between the first and second resection. Patients were considered to have repeat resection if their repeat resection occurred by that time point, and were considered to not have repeat resection if they did not undergo a repeat resection or if their repeat resection occurred after the specified time period (either 8 or 14 months from initial surgery). Patients who died before 8 and 14 months were excluded from these plots as per landmark methodology. ${ }^{2}$ Patients who had a repeat resection by 8 months $(A ; n=43)$ had a median OS of 8.1 months ( $95 \%$ Cl $5.1-11.0$ months), compared with 13.9 months (95\% Cl 11.8-17.7 months) for patients who did not undergo a repeat resection by 8 months $(n=94)$. Patients who had a repeat resection by 14 months $(B ; n=41)$ had a median OS of 9.0 months $(95 \% \mathrm{Cl} 4.7-11.7$ months), compared with a median of 12.0 months $(95 \% \mathrm{Cl} 7.9-15.9$ months) for those who did not undergo a repeat resection by 14 months $(n=58)$. This figure is an illustrative tool for the formal time-dependent analysis, and cannot be used to make inference. Figure is available in color online only.

had a lower risk of death (HR $0.62,95 \%$ CI $0.43-0.90$, $\mathrm{p}=0.01)$ compared with those without repeat resection. However, once we incorporated timing of resection, the relationship between repeat resection and OS reversed. $\mathrm{Pa}-$ tients who had repeat resection had a higher risk of death (HR 2.19, 95\% CI 1.47-3.28, p < 0.001) compared with those who did not undergo repeat resection, even after controlling for EOR, age, eloquent status, multifocal status, initial KPS, adjuvant treatment, and sex (Table 3).

\section{Post Hoc: Factors Associated With Repeat Resection}

Younger age (HR 0.98, 95\% CI 0.96-1.00, $\mathrm{p}=0.050$ ), higher initial KPS score (HR 1.03, 95\% CI 1.01-1.05, p = 0.009 ), and greater EOR at initial surgery (HR 9.52, 95\% CI $2.29-39.52, \mathrm{p}=0.002$ ) were associated with repeat resection. Eloquently located tumors also trended toward association with repeat resection (HR $0.67,95 \%$ CI $0.44-$ $1.02, p=0.06)$. No significant associations were found for sex $(\mathrm{p}=0.45)$, race $(\mathrm{p}=0.81)$, or multifocal status $(\mathrm{p}=$ $0.09)$.

\section{Discussion}

In our study, when timing of repeat resection was ig- nored, we found a similar HR to previously published findings. ${ }^{8,32}$ Once timing of repeat resection was incorporated with time-dependent methods, the relationship between OS and repeat resection reversed. This remained even after controlling for known predictors of OS, including eloquent status, multifocal status, age, sex, EOR, and adjuvant treatment. We believe that the often-reported benefit of repeat resection may have resulted from methodology that ignored the timing of repeat resection.

We identified two studies that used time-dependent methods ${ }^{13,16}$ and one study that used landmark methods ${ }^{11}$ when assessing the relationship between repeat resection and OS in glioblastoma. Filippini and colleagues ${ }^{13}$ assessed factors associated with survival after recurrence and found no significant difference in risk for those who had second surgery within or after 9 months. However, their study included patients who underwent only initial diagnostic biopsy, and it was unclear whether repeat resection also included diagnostic biopsies. In the second study with time-dependent methods, Fogh and colleagues ${ }^{16}$ ex- $^{-}$ amined the survival benefits of hypofractionated stereotactic radiation therapy (HSRT) on patients with recurrent Grade III and IV gliomas. Similar to the study of Filippini et al., ${ }^{13}$ their sample also included patients who underwent 
TABLE 3. Univariable and multivariable Cox proportional hazards regression using time-dependent covariates

\begin{tabular}{|c|c|c|c|c|}
\hline \multirow[b]{2}{*}{ Variable } & \multicolumn{2}{|c|}{ Univariable } & \multicolumn{2}{|c|}{ Multivariable } \\
\hline & $\mathrm{HR}(95 \% \mathrm{Cl})$ & p Value & $\mathrm{HR}(95 \% \mathrm{Cl})$ & p Value \\
\hline Age & $1.03(1.01-1.05)$ & 0.008 & $1.02(1.00-1.04)$ & 0.02 \\
\hline \multicolumn{5}{|l|}{ Eloquent region tumor* } \\
\hline Yes & $1.54(1.08-2.20)$ & 0.02 & $1.71(1.15-2.55)$ & 0.009 \\
\hline No & - & & & \\
\hline \multicolumn{5}{|l|}{ Sex } \\
\hline Female & $0.67(0.45-0.99)$ & 0.04 & $0.65(0.43-0.97)$ & 0.04 \\
\hline Male & - & & & \\
\hline \multicolumn{5}{|l|}{ Multifocal $^{*}$} \\
\hline Yes & $1.86(1.14-3.03)$ & 0.01 & $1.55(0.86-2.79)$ & 0.15 \\
\hline No & - & & & \\
\hline PostOp1 treatment (ordinal)* & $0.54(0.40-0.73)$ & $<0.001$ & $0.64(0.46-0.88)$ & 0.007 \\
\hline Initial KPS score & $0.98(0.97-1.00)$ & 0.03 & $0.99(0.98-1.01)$ & 0.46 \\
\hline \multicolumn{5}{|l|}{ Repeat resection* } \\
\hline Yes & $2.02(1.40-2.91)$ & $<0.001$ & $2.19(1.47-3.28)$ & $<0.001$ \\
\hline No & - & & & \\
\hline EOR (continuous)* & $0.15(0.06-0.37)$ & $<0.001$ & $0.38(0.11-1.25)$ & 0.11 \\
\hline \multicolumn{5}{|l|}{ PostOp1 chemotherapy* } \\
\hline Yes & $0.30(0.17-0.56)$ & $<0.001$ & & \\
\hline No & - & & & \\
\hline \multicolumn{5}{|l|}{ PostOp1 radiation* } \\
\hline Yes & $0.31(0.17-0.56)$ & $<0.001$ & & \\
\hline No & - & & & \\
\hline \multicolumn{5}{|l|}{ Initial KPS score (grouped) } \\
\hline$\leq 70$ & $1.25(0.82-1.90)$ & 0.30 & & \\
\hline$>70$ & - & & & \\
\hline EOR initial surgery & $0.30(0.11-0.81)$ & 0.02 & & \\
\hline \multicolumn{5}{|l|}{ EOR (grouped 97\%)* } \\
\hline$\geq 97 \%$ & $0.59(0.39-0.88)$ & 0.009 & & \\
\hline$<97 \%$ & - & & & \\
\hline
\end{tabular}

Boldface type indicates statistical significance.

* Time-dependent covariate.

only biopsy. Surgery after HSRT was treated as a timedependent covariate and second resection before HSRT was treated as a standard covariate. Neither surgery after HSRT nor before showed an association with survival. Clarke et al. ${ }^{11}$ used a landmark method in a retrospective analysis, but patients were drawn from those enrolled in North American Brain Tumor Consortium prospective trials. They, too, failed to find a benefit to repeat resection for patients from older or newer studies. In brief, and despite critical differences in methodology and patient selection, whenever timing of repeat resection was accounted for in the analysis, no survival benefit was found.

Our findings raise the possibility that patients with poorer risk factors were more likely to be offered and to receive repeat resection, such that the differences in survival may be due to underlying risk factors rather than to repeat resection itself. ${ }^{15}$ Despite these patients undergoing treatment consistently by the same physicians who adhered to tumor board recommendations, due to the retro- spective nature of our study, selection bias cannot be ruled out. Higher initial KPS score and greater EOR at initial resection were associated with repeat resection. Younger age was also associated with repeat resection, and older age is a known risk factor for reduced survival..$^{22}$ These findings suggest that patients with repeat resection had characteristics associated with a lower risk of death and may have been able to better tolerate repeat resection, which aligns with findings from the heart transplant literature. ${ }^{2,12,23}$ Nonetheless, once we controlled for these factors, the reversed association with OS persisted. Of note, we were missing KPS score at repeat resection among other health measurements during follow-up, which may change between initial and repeat resection. It is also possible that patients who had repeat resection had higher rates of neurological symptoms, which required palliation, but ultimately reduced their survival.

Similarly, our study was not designed to answer why repeat resection was associated with a decreased survival 
probability. Explanations for our findings include surgical complications, delayed chemotherapy, or particularly aggressive tumor phenotypes, which required, but did not benefit from surgery. Only 1 patient died within 30 days of repeat resection, so it is unlikely that morbidity from surgery affected survival. However, we did not have comprehensive complications data. Additionally, many studies suggest underlying molecular variations, including methylation status and $I D H-1$ mutations, are associated with tumor aggressiveness and survival. . $9,27,28,34$ Unfortunately, our molecular and genetic data were limited. We recommend that future studies collect and incorporate these data to further explore these possibilities.

Importantly, our findings do not necessarily indicate that repeat resection is without benefit to individual patients or to a certain subgroup of patients. Surgery may improve quality of life or functional status by reducing tumor burden ${ }^{1}$ and/or reducing dependence on steroids. This is particularly critical as more immunotherapy regimens become available to patients with glioblastoma, as these mandate minimal or no steroid intake. Furthermore, it may allow patients to be eligible for clinical trials of novel treatment as tumor size and functional status are frequently eligibility criteria (e.g., clinical trial nos. NCT01491893 and NCT00209989; www.clinicaltrials.gov).

Despite the novelty of our findings, our analysis is not without limitations. Our study was retrospective and, as such, was subject to inherent limitations such as treatment heterogeneity, sampling bias, and incomplete data. For instance, not all patients are eligible to receive repeat resection and those patients who are worse off may be the ones who receive repeat resection, which creates a sampling bias. This bias is an inherent part of retrospective repeat resection studies unless it can be studied in a randomized prospective trial. Furthermore, we chose not to analyze patients with craniotomies performed at outside institutions because imaging was frequently missing. This limits the generalizability of our findings. Additionally, although we had a considerable number of events, it would still benefit the literature to have this assessed on a larger cohort. For example, too few patients received third or fourth resections to assess the benefit of multiple repeat resections on OS.

\section{Conclusions}

Our study demonstrates that incorporating the timing of repeat resection in patients with glioblastoma using time-dependent methods reverses the protective effect of repeat resection on OS, suggesting repeat resection may not benefit OS in all patients. Our findings are similar to theoretical findings and results in other medical fields where time-dependent methods were previously employed. This study establishes a foundation for analyzing repeat resection with survival, and will likely initiate a healthy debate on the survival benefits of repeat resection. However, future work must build upon this foundation to gain a better understanding of this topic, particularly concerning survival benefits in specific subgroups, including those defined by molecular data. Our additional recommendations involve improved data capture including molecular tumor information, development of algorithms to determine eligibility for repeat resection, assessment of additional benefits of repeat resection (such as reduction of symptom burden and enhanced quality of life), and more rigorous statistical analyses applied to these complicated research questions that cannot be easily assessed in randomized clinical trials.

\section{Acknowledgments}

We acknowledge the support of the MSKCC Biostatistics Core (NIH grant no. P30 CA008748). We also thank Mithat Gonen, PhD, and Eli Diamond, MD, for their comments on this paper.

\section{References}

1. Ammirati M, Vick N, Liao YL, Ciric I, Mikhael M: Effect of the extent of surgical resection on survival and quality of life in patients with supratentorial glioblastomas and anaplastic astrocytomas. Neurosurgery 21:201-206, 1987

2. Anderson JR, Cain KC, Gelber RD: Analysis of survival by tumor response. J Clin Oncol 1:710-719, 1983

3. Azizi A, Black P, Miyamoto C, Croul SE: Treatment of malignant astrocytomas with repetitive resections: a longitudinal study. Isr Med Assoc J 3:254-257, 2001

4. Azoulay M, Santos F, Souhami L, Panet-Raymond V, Petrecca K, Owen S, et al: Comparison of radiation regimens in the treatment of Glioblastoma multiforme: results from a single institution. Radiat Oncol 10:106, 2015

5. Beyersmann J, Gastmeier P, Wolkewitz M, Schumacher M: An easy mathematical proof showed that time-dependent bias inevitably leads to biased effect estimation. J Clin Epidemiol 61:1216-1221, 2008

6. Beyersmann J, Wolkewitz M, Schumacher M: The impact of time-dependent bias in proportional hazards modelling. Stat Med 27:6439-6454, 2008

7. Bloch O, Han SJ, Cha S, Sun MZ, Aghi MK, McDermott MW, et al: Impact of extent of resection for recurrent glioblastoma on overall survival: clinical article. J Neurosurg 117:1032-1038, 2012

8. Chaichana KL, Zadnik P, Weingart JD, Olivi A, Gallia GL, Blakeley J, et al: Multiple resections for patients with glioblastoma: prolonging survival. J Neurosurg 118:812-820, 2013

9. Chaiteerakij R, Petersen GM, Bamlet WR, Chaffee KG, Zhen DB, Burch PA, et al: Metformin use and survival of patients with pancreatic cancer: a cautionary lesson. J Clin Oncol 34:1898-1904, 2016

10. Chen MW, Morsy AA, Liang S, Ng WH: Re-do craniotomy for recurrent grade IV glioblastomas: impact and outcomes from the National Neuroscience Institute Singapore. World Neurosurg 87:439-445, 2016

11. Clarke JL, Ennis MM, Yung WKA, Chang SM, Wen PY, Cloughesy TF, et al: Is surgery at progression a prognostic marker for improved 6-month progression-free survival or overall survival for patients with recurrent glioblastoma? Neuro Oncol 13:1118-1124, 2011

12. Crowley J, Hu M: Covariance analysis of heart transplant survival data. J Am Stat Assoc 72:27-36, 1977

13. Filippini G, Falcone C, Boiardi A, Broggi G, Bruzzone MG, Caldiroli D, et al: Prognostic factors for survival in 676 consecutive patients with newly diagnosed primary glioblastoma. Neuro Oncol 10:79-87, 2008

14. Fine JP, Gray RJ: A proportional hazards model for the subdistribution of a competing risk. J Am Stat Assoc 94:496509, 1999

15. Fisher LD, Lin DY: Time-dependent covariates in the Cox proportional-hazards regression model. Annu Rev Public Health 20:145-157, 1999 
16. Fogh SE, Andrews DW, Glass J, Curran W, Glass C, Champ $\mathrm{C}$, et al: Hypofractionated stereotactic radiation therapy: an effective therapy for recurrent high-grade gliomas. J Clin Oncol 28:3048-3053, 2010

17. Gittleman H, Lim D, Kattan MW, Chakravarti A, Gilbert MR, Lassman AB, et al: An independently validated nomogram for individualized estimation of survival among patients with newly diagnosed glioblastoma: NRG Oncology RTOG 0525 and 0825. Neuro Oncol, 2016

18. Guyotat J, Signorelli F, Frappaz D, Madarassy G, Ricci AC, Bret P: Is reoperation for recurrence of glioblastoma justified? Oncol Rep 7:899-904, 2000

19. Helseth R, Helseth E, Johannesen TB, Langberg CW, Lote $\mathrm{K}$, Rønning P, et al: Overall survival, prognostic factors, and repeated surgery in a consecutive series of 516 patients with glioblastoma multiforme. Acta Neurol Scand 122:159-167, 2010

20. Iacobelli S: Suggestions on the use of statistical methodologies in studies of the European Group for Blood and Marrow Transplantation. Bone Marrow Transplant 48 (Suppl 1):S1-S37, 2013

21. Jatoi I, Anderson WF, Jeong JH, Redmond CK: Breast cancer adjuvant therapy: time to consider its time-dependent effects. J Clin Oncol 29:2301-2304, 2011

22. Lacroix M, Abi-Said D, Fourney DR, Gokaslan ZL, Shi W, DeMonte F, et al: A multivariate analysis of 416 patients with glioblastoma multiforme: prognosis, extent of resection, and survival. J Neurosurg 95:190-198, 2001

23. Mantel N, Byar DP: Evaluation of response-time data involving transient states: an illustration using heart-transplant data. J Am Stat Assoc 69:81-86, 1974

24. Mason WP, Maestro RD, Eisenstat D, Forsyth P, Fulton D, Laperrière $\mathrm{N}$, et al: Canadian recommendations for the treatment of glioblastoma multiforme. Curr Oncol 14:110-117, 2007

25. Oppenlander ME, Wolf AB, Snyder LA, Bina R, Wilson JR, Coons SW, et al: An extent of resection threshold for recurrent glioblastoma and its risk for neurological morbidity. J Neurosurg 120:846-853, 2014

26. Ostrom QT, Gittleman H, Fulop J, Liu M, Blanda R, Kromer C, et al: CBTRUS Statistical Report: Primary Brain and Central Nervous System Tumors Diagnosed in the United States in 2008-2012. Neuro Oncol 17 (Suppl 4):iv1-iv62, 2015

27. Panosyan EH, Lasky JL, Lin HJ, Lai A, Hai Y, Guo X, et al: Clinical aggressiveness of malignant gliomas is linked to augmented metabolism of amino acids. J Neurooncol 128:57-66, 2016

28. Rao A, Rao G, Gutman DA, Flanders AE, Hwang SN, Rubin DL, et al: A combinatorial radiographic phenotype may stratify patient survival and be associated with invasion and proliferation characteristics in glioblastoma. J Neurosurg 124:1008-1017, 2016

29. Ringel F, Pape H, Sabel M, Krex D, Bock HC, Misch M, et al: Clinical benefit from resection of recurrent glioblastomas: results of a multicenter study including 503 patients with recurrent glioblastomas undergoing surgical resection. Neuro Oncol 18:96-104, 2016

30. Simon R, Makuch RW: A non-parametric graphical representation of the relationship between survival and the occurrence of an event: application to responder versus non-responder bias. Stat Med 3:35-44, 1984

31. Stupp R, Mason WP, van den Bent MJ, Weller M, Fisher $\mathrm{B}$, Taphoorn MJ, et al: Radiotherapy plus concomitant and adjuvant temozolomide for glioblastoma. $\mathbf{N}$ Engl J Med 352:987-996, 2005

32. Tully PA, Gogos AJ, Love C, Liew D, Drummond KJ, Morokoff AP: Reoperation for recurrent glioblastoma and its association with survival benefit. Neurosurgery 79:678-689, 2016

33. van Walraven C, Davis D, Forster AJ, Wells GA: Timedependent bias was common in survival analyses published in leading clinical journals. J Clin Epidemiol 57:672-682, 2004

34. Wang W, Zhang L, Wang Z, Yang F, Wang H, Liang T, et al: A three-gene signature for prognosis in patients with MGMT promoter-methylated glioblastoma. Oncotarget 7:6999169999, 2016

\section{Disclosures}

The authors report no conflict of interest concerning the materials or methods used in this study or the findings specified in this paper.

\section{Author Contributions}

Conception and design: Panageas, Goldman, Reiner. Acquisition of data: Goldman, Hovinga, Esquenazi, Tabar. Analysis and interpretation of data: Panageas, Goldman, Reiner, Tabar. Drafting the article: Panageas, Goldman, Reiner. Critically revising the article: all authors. Reviewed submitted version of manuscript: all authors. Approved the final version of the manuscript on behalf of all authors: Panageas. Statistical analysis: Panageas, Goldman, Reiner. Administrative/technical/material support: Tabar. Study supervision: Panageas, Tabar.

\section{Correspondence}

Katherine S. Panageas: Memorial Sloan Kettering Cancer Center, New York, NY.panageak@mskcc.org. 